\title{
Structural and compositional changes in erythrocyte membrane of obese compared to normal-weight adolescents
}

\author{
Javier S. Perona ${ }^{1 a}$, Emilio González-Jiménez ${ }^{2 a}$, María J. Aguilar-Cordero ${ }^{3}$, Antonio Sureda ${ }^{4}$ and Francisca $^{2}$ \\ Barceló $^{5^{*}}$ \\ ${ }^{1}$ Instituto de la Grasa, Consejo Superior de Investigaciones Científicas (IG-CSIC), Av. Padre García Tejero, 4, E- \\ 41012 Sevilla, Spain. \\ ${ }^{2}$ Departamento de Enfermería. Facultad de Enfermería (Campus de Melilla). Universidad de Granada. C/ \\ Santander, 1, E-52071 Melilla, Spain. \\ ${ }^{3}$ Departamento de Enfermería. Facultad de Ciencias de la Salud. Universidad de Granada. Av. de Madrid s/n, E- \\ 18071 Granada, Spain.
}

${ }^{4}$ Research Group on Community Nutrition and Oxidative Stress, University of the Balearic Islands, E-07122 Palma de Mallorca, Spain.

${ }^{5}$ Clinical and Translational Research Group, University of the Balearic Islands, E-07122 Palma de Mallorca, Spain.

"Correspondence: Francisca Barceló. Departamento de Biología Fundamental y Ciencias de la Salud. University of the Balearic Islands. Ctra. Valldemosa, Km 7.5. E-07122, Palma de Mallorca. Spain. Phone: +34971173149. Fax: +34971173184. E-mail: francisca.barcelo@,uib.es

\footnotetext{
1a These authors contributed equally to this work. 1
} 
Abbreviations: AChE, acetylcholinesterase enzyme; BMI, Body mass index; Cho, cholesterol; L $\alpha$, lamellar liquid crystalline phase; d-value, repeat distance of a lamellar phase; MDA, malondialdehyde; MLV, multilamellar lipid vesicles; MUFA, monounsaturated fatty acids; PL, phospholipid; PUFA, polyunsaturated fatty acids; SAXS, Small-Angle Synchrotron radiation X-ray scattering; SFA, saturated fatty acid; UFA, unsaturated fatty acids; UI, unsaturation index; WAXS, Wide-Angle Synchrotron radiation X-ray scattering; XRD, X-ray diffraction. 


\begin{abstract}
Unhealthy dietary habits are key determinants of obesity in adolescents. Assuming that dietary fat profile influences membrane lipid composition, the aim of this study was to analyse structural changes in the erythrocyte membrane of obese compared to normal-weight adolescents. The study was conducted in a group of 11 obese and 11 normal-weight adolescent subjects. The lipid profile, lipid peroxidation and acetylcholinesterase enzyme (AChE) activity were analysed by conventional methods. The structural properties of reconstituted erythrocyte membrane were characterized by X-ray diffraction. Erythrocyte membrane from obese adolescents had a lipid profile characterized by a higher cholesterol/phospholipid ratio, an increase in SFA and a decrease in MUFA and n-6 PUFA fatty acid concentration. Differences in the lipid content were associated with changes in the structural properties of reconstituted membranes and the oxidative damage of erythrocyte membrane. The lower oxidative level shown in the obese group $(0.15 \pm 0.04 \mathrm{vs} .0 .20 \pm 0.06 \mathrm{nmol} / \mathrm{mg}$ for conjugated dienes concentrations and $2.43 \pm 0.25$ vs. $2.83 \pm 0.31 \mathrm{nmol} / \mathrm{mg}$ protein for malondihaldeide levels) was related to a lower unsaturation index (UI). These changes in the membrane structure properties were accompanied by a lower AChE activity (1.64 \pm 0.13 vs. $1.91 \pm 0.24 \mathrm{nmol} \mathrm{AChE} / \mathrm{min} \cdot \mathrm{mg}$ protein) in the obese group. The consequences of unhealthy dietary habits in adolescents are reflected in the membrane structural properties and may influence the membrane associated protein activities and functions.
\end{abstract}

Keywords: Acetilcholiesterase activity; adolescents; erythrocyte membrane structure; lipid peroxidation; obesity. 


\section{Introduction}

The number of obese children has grown considerably in developed countries in recent years (Sinha et al. 2009). Due to the importance of the problem, there is growing research on the relationship between lifestyle and obesity in adolescence (Dietz 1998; Collins et al. 2010). In the teenager population, genes play a role like in adults (Jacobsson et al. 2012). However, the dietary lipid profile is also a factor that affects body weight gain inducing obesity and associated diseases. Thus, the influence of an obesogenic environment is widely accepted as a significant cause for the increase of weight in the adolescent population (Symonds et al. 2009).

It has been demonstrated that dietary fat intake influences the fatty acid composition of cell membranes (Barceló et al. 2009). Changes in the fatty acid profiles have an impact on the membrane structural characteristics and may influence membrane protein functions that take part in relevant physiological activities. For example, alterations in the cell membrane lipid composition of hypertensive subjects affect signalling proteins that participate in the control of blood pressure (Escriba et al. 2003). On the other hand, lipid peroxidation under conditions of oxidative stress can also contribute to alterations in membrane structure/function (Jacob et al. 2005). In obese patients, a high metabolic pro-oxidant status of erythrocytes alters membrane properties and has been associated to obesity-related pathologies (Cazzola et al. 2004). It was argued that dietary fat might influence the cellular metabolism through an effect on membrane lipid composition, but the study did not examine in detail how the diet affects the membrane structure in the context of the obesity development (Pan et al. 1994). There is a lack of experimental data relating cell metabolism and membrane structure. However, if cell membrane properties are affected by environmental factors, the diet profile of adolescents can influence the structural membrane characteristics and associated protein functions. Thus, the global result might be a cellular metabolic rearrangement that accompanies the obesity. This possibility might represent an additional effect on the disease to be considered.

The aim of this study was to analyse the structural properties of erythrocyte membrane from obese and normal-weight adolescent subjects and correlate them with the oxidative effect derived from the free radical damage. The acetylcholinesterase enzyme (AChE) activity was also examined, as a membrane protein and a marker of the non-neuronal cholinergic system in human erythrocytes. Our findings provide information 
regarding (i) the lipid profile, lipid peroxidation and AChE activity of erythrocyte membranes from obese adolescents and (ii) how differences in the lipid composition are associated with changes in the structural properties of reconstituted erythrocytes membranes from obese and normal-weight adolescents. 


\section{Materials and Methods}

\section{Materials}

Lipid standards, cholesterol, 1,2-dipalmitoyl-sn-glycero-3-phosphatidylethanolamine, 1,2-Diacyl-sn-glycero-3phosphocholine, 1,2-Diacyl-sn-glycero-3-phospho-L-serine, N-Acyl-4-sphingenyl-1-O-phosphorylcholine and lisophosphatidylcholine and the chemical compounds N-(2-Hydroxy ethyl) piperazine-N'-(2-ethanesulfonic acid) sodium salt (Hepes), acetylthiocholine iodide, 5,5'-dithiobis-2-nitrobenzoic acid and 5,5'-dithiobis-2nitrobenzoic acid were purchased from Sigma-Aldrich (Madrid, Spain). Solvents HPLC-grade were from Romil (Cambridge, UK). Lichrosphere diol column was from Merck (Darmstadt, Germany) and Supelcowax 10 capillary silica column was from Sigma-Aldrich (Madrid, Spain).

\section{Study design}

The present work is a part of a major project designed to evaluate the clinical and molecular parameters that characterize the prevalence of obesity among adolescents in the area of the province of Granada (Spain) (González-Jiménez et al. 2012). Its aim was to analyse the structural and compositional properties and oxidative damage of erythrocyte membrane of obese and normal-weight adolescents. 22 subjects (12-16 years) without a history of metabolic disorders were recruited from the public school in the city of Guadix (Granada, Spain). All participants belonged to the same community (a city of 21000 inhabitants), attended the same high-school and had similar dietary and cultural habits, physical activity and socio-economic level. Subjects were classified as obese, using the body mass index (BMI) score and following the indications of the Obesity Task Force, according to Cole et al. (2000). 11 subjects were obese (BMI $>30 \mathrm{Kg} / \mathrm{m}^{2}$ and percentile $\left.>97\right)$ and 11 normalweight $\left(\mathrm{BMI}=20-25 \mathrm{Kg} / \mathrm{m}^{2}\right.$ and a range percentile 5-75). The volunteers' parents gave written informed consent to participate in the study following the protocol approved by the Institutional Committee on Human Research (Hospital San Cecilio, Granada, Spain). All procedures were in accordance with the Institutional and National ethical standards for human experimentation and the Helsinki declaration of 1975 (revised in 2000). 
Blood sample collection and erythrocyte membrane preparation

Venus blood samples were taken via a cubital vein catheter after overnight fasting and used for erythrocyte membrane preparations. Blood samples were collected in presence of EDTA and immediately processed in order to reduce oxidation processes. Erythrocyte membranes were isolated by centrifugation as described previously (Ruíz-Gutiérrez et al. 1996). Membrane pellets were washed twice with $110 \mathrm{mM} \mathrm{MgCl}_{2}$, sedimented at $1750 \mathrm{~g}$ for $5 \mathrm{~min}$ and immediately stored at $-80^{\circ} \mathrm{C}$ under nitrogen until use.

\section{Lipid classes and fatty acid methyl esters analysis}

Membrane lipids were extracted with chloroform:methanol (2:1, v:v), according to the method of Folch et al. (1957). Lipid classes were analysed by HPLC (2690 Alliance, Waters Co., Milford, MA), as described previously (Perona et al., 2004), using a Lichrosphere diol column ( 250 x 4.6 mm, $5-\mu \mathrm{m}$ particle size, Merck) and a light-scattering detector (ELSD 2420 Waters Co., Milford, MA). Fatty acid methyl esters were quantified with a Hewlett-Packard 5890 series II gas chromatograph equipped with a flame ionization detector (HewlettPackard Co, Avondale, USA) and a Supelcowax 10 capillary silica column $(60 \mathrm{~m}$ and $0.25 \mathrm{~mm}$ internal diameter, Sulpelco Co, Bellefonte, USA), according to recognized protocols (Ruiz-Gutierrez et al. 1992).

\section{Erythrocyte membrane oxidation analysis}

Conjugated dienes measurement. The concentration of erythrocyte-conjugated dienes was measured according to the method of Reilly and Aust (1999). Membrane lipid extract (1 g/subject) dissolved in $1.5 \mathrm{ml}$ cyclohexane was quantified by measuring the absorbance at $233 \mathrm{~nm}$ using an extinction coefficient of $2.52 \times 10^{4} \mathrm{M}^{-1} \mathrm{~cm}^{-1}$. All data are mean values of three independent experiments performed in duplicates.

Malondialdehyde (MDA) assay. Membrane lipid peroxidation was analysed by a colorimetric assay kit (Calbiochem, SanDiego, CA, USA) to measure MDA levels. Experimental procedures were performed according to the manufacturer's instructions. MDA-erythrocyte samples and MDA standards of known 
concentration were placed in glass tubes containing the chromogenic agent methyl-2-phenylindole $(10.3 \mathrm{mM})$ in acetonitrile:methanol $(3: 1, \mathrm{v}: \mathrm{v})$. After the addition of $\mathrm{HCl} 12 \mathrm{~N}$, samples were incubated at $45{ }^{\circ} \mathrm{C}$ for $1 \mathrm{~h}$. MDAerythrocyte levels were measured at $586 \mathrm{~nm}$ and calibrated against the standard curve. All data are mean values of three independent experiments.

MDA-protein adducts assay. MDA-protein adducts were determined by an enzyme immunoassay (Cell Biolabs, Inc.). Erythrocyte samples $(10 \mu \mathrm{g}$ protein $/ \mathrm{ml})$ were probed with an anti-MDA antibody, followed by a horseradish peroxidase conjugated secondary antibody. The MDA protein adducts content was quantified from a MDA-BSA standard curve.

\section{Acetylcholinesterase activity assay}

The erythrocyte acetylcholinesterase (AChE) activity was quantified by a spectrophotometric method based on the enzymatic hydrolysis of acetylthiocholine iodide (Ellman et al. 1961). Measurements were performed at 37 ${ }^{\circ} \mathrm{C}$ in hemolysate samples (100 $\mu \mathrm{g}$ protein). Enzymatic activity was analysed in $1 \mathrm{M}$ Tris-HCl buffer $\mathrm{pH} 7.4$, containing $5 \mathrm{mM}$ EDTA and $0.5 \mathrm{mM} \mathrm{5,5'-dithiobis-2-nitrobenzoic} \mathrm{acid.} \mathrm{The} \mathrm{reaction} \mathrm{was} \mathrm{initiated} \mathrm{by} \mathrm{the} \mathrm{addition}$ of $10 \mathrm{mM}$ acetylthiocholine iodide and monitored at $412 \mathrm{~nm}$. Enzyme activity data are mean values of two independent experiments.

\section{Erythrocyte model membrane preparation}

Total lipids of erythrocyte membranes, extracted with chloroform/methanol (2:1, v:v) (Folch et al. 1957), were used to reconstitute erythrocyte model membranes. Multilamellar lipid vesicles (MLV) 15\% (w/w) were prepared in $10 \mathrm{mM}$ Hepes, $100 \mathrm{mM} \mathrm{NaCl}, 1 \mathrm{mM}$ EDTA, pH 7.4 (Hepes buffer), following established procedures (Funari et al. 2003). Lipid mixtures were hydrated, thoroughly homogenized with a pestle-type minihomogenizer (Sigma) and vortexed to obtain homogeneous samples. The suspensions were submitted to seven temperature cycles (heated up to $70^{\circ} \mathrm{C}$ and cooled down to $-20^{\circ} \mathrm{C}$ ). Samples were stored under argon at $-80^{\circ} \mathrm{C}$ until use. They were equilibrated at $4^{\circ} \mathrm{C}$ during 24 hours before the analysis. 
$X$-ray diffraction analysis

Small- and Wide-Angle Synchrotron radiation X-ray scattering (SAXS and WAXS) experiments were conducted on the Soft Condensed Matter beamline A2 at the storage ring Doris III of HASYLAB (DESY, Hamburg, Germany) using radiation of $0.15 \mathrm{~nm}$ wavelength. The scattering patterns or images were collected by a MAR $1652 \mathrm{D}$ detector. The samples were loaded into a temperature-controlled holder, heated from 10 to $60^{\circ} \mathrm{C}$ and then cooled down to $10^{\circ} \mathrm{C}$ at a scan rate of $1^{\circ} \mathrm{C} / \mathrm{min}$. The system was equilibrated $10 \mathrm{~min}$ at each temperature before measurements. For SAXS data analysis, a circular sector from each image was integrated using A2tool, an in-house written software (by A. Rothkirch), producing 1D scattering curves. The WAXS data analysis was as described previously (Funari et al. 2003). The positions of the observed peaks were converted into distances, $d$, after calibration with the standards rat tendon tail and poly-(ethylene therephtalate) for the SAXS and WAXS regions, respectively. Interplanar distances, $d_{h k l}$, were calculated according to the equation:

$$
s=1 / d_{h k l}=(2 \sin \theta) / \lambda
$$

where $s$ represent the scattering vector, $2 \theta$ the scattering angle, $\lambda(0.15 \mathrm{~nm})$ the X-ray wavelength and $h k l$ are the Miller indexes of the scattering planes.

\section{Statistical analysis}

The analysis was performed with the software package SPSS Statistics (SPSS 21.0, Chicago, IL, USA). The Shapiro-Wilk W-test was applied to assess the normal distribution of the experimental data. The statistical significance was analysed by the unpaired Student's t-test and Pearson's coefficient for correlations. Data are reported as mean value \pm standard deviation (SD). Differences were considered statistically significant at $\mathrm{P} \leq$ 0.05 . 


\section{Results}

Membrane lipid composition

The lipid composition of erythrocyte membrane from the obese and normal-weight groups is shown in Table 1. The cholesterol (Cho), but not the phospholipid (PL) content, was significantly higher in obese subjects compared to normal-weight participants. Therefore, the Cho/PL ratio was superior in the obese group. Table 2 illustrates the fatty acid composition. Differences were observed in the percentage of saturated (SFA: palmitic and stearic), monounsaturated (MUFA: oleic) and n-6 polyunsaturated (PUFA: linoleic and arachidonic) fatty acids between obese and normal-weight adolescents subjects. In the obese group, the SFA content was considerably higher. There was an important decrease in the n-6 PUFA and the n-9 MUFA percentage while the n-3 fatty acid content and the n-6/n-3 ratio were scarcely altered. The significant lower n-6/n-3 ratio shown by the obese participants was mostly attributable to a minor amount of linoleic and arachidonic acids. The global effect of these changes was a reduction in the membrane unsaturation index (UI) that characterized the erythrocyte membrane of obese adolescents.

\section{Membrane oxidation}

The concentrations of erythrocyte-conjugated dienes and MDA levels in the obese and normal-weight groups are depicted in Fig. 1. Obese subjects had lower erythrocyte-conjugated dienes $(0.15 \pm 0.04$ vs. $0.20 \pm 0.06$ $\mathrm{nmol} / \mathrm{mg}, \mathrm{P}=0.040)$ and erythrocyte-MDA $(2.43 \pm 0.25$ vs. $2.83 \pm 0.31 \mathrm{nmol} / \mathrm{mg}$ protein, $\mathrm{P}=0.003)$ levels. Both markers correlated $(\mathrm{r}=0.50 ; \mathrm{P}=0.02)$. The MDA-protein adducts assay did not show differences between both adolescent groups (data not shown). Correlation analysis between the lipid peroxidation data and the membrane fatty acid component were also performed. Conjugated dienes values correlated positively with palmitie $(\mathrm{r}=$ $0.63 ; \mathrm{P}=0.04)$ and linoleic acid concentrations $(\mathrm{r}=0.75 ; \mathrm{P}=0.01)$ in the control group and negatively with stearic $(\mathrm{r}=0.75 ; \mathrm{P}=0.01)$ and oleic $(\mathrm{r}=0.74 ; \mathrm{P}=0.02)$ acids in the obese group. In turn, MDA marker was significantly related with PUFA $(r=0.41 ; \mathrm{P}=0.02)$, SFA/PUFA $(r=0.51 ; \mathrm{P}=0.03)$ and $16: 0$ to $18: 2$ ratio $(\mathrm{r}=$ $0.62 ; \mathrm{P}=0.02$ ) in both groups. Linoleic acid was revealed as a key fatty acid regarding to peroxidation via direct correlation with peroxidation markers. 


\section{Erythrocyte AChE enzyme activity}

The AChE activity was measured as an indicator of the non-neuronal cholinergic system. The enzyme activity levels are shown in Fig. 2. The obese group showed lower AChE activity compared to the normal-weight group $(1.64 \pm 0.13 v s .1 .91 \pm 0.24 \mathrm{nmol} \mathrm{AChE} / \mathrm{min} \cdot \mathrm{mg}$ protein, $\mathrm{P}=0.007)$.

\section{Supramolecular organization and lipid phase behaviour of reconstituted erythrocyte model membranes}

Erythrocyte membranes reconstituted with the total cell lipid extract isolated from each subject were analysed by X-ray diffraction. Fig. 3A illustrates that membranes displayed a clear X-ray diffraction pattern composed by a lamellar $\mathrm{L}_{\alpha}$ phase. Each sample had a particular pattern that could be taken as a fingerprint of the erythrocyte membranes. Fig. 3B depicts representative peak diffraction plots of the MLV (15\% w/w) reconstituted erythrocyte membranes. Obese and normal-weight groups had comparable structural characteristics. The SAXS patterns showed two main diffraction peaks, corresponding to the first and the second order of the lamellar $\mathrm{L}_{\alpha}$ phase, and a third peak at $\mathrm{s}=0.289 \mathrm{~nm}^{-1}$ assigned to a crystalline Cho structure. All samples exhibited reversible thermotropic behaviour on cooling. In some patterns, there were indications of a possible coexistence of two structural systems in the range temperatures $40-50{ }^{\circ} \mathrm{C}$ (probably two lamellar phases), but the weak diffraction peaks did not allow to draw any conclusion. Several controls (e.g. samples C-2, C-18 and C-19) displayed a peculiar diffraction pattern, presenting coexistence of two $\mathrm{L}_{\alpha}$ phases at low temperature that melted into one at $20{ }^{\circ} \mathrm{C}$ and then the system proceeded as a simple lamellar phase. The diffraction peaks in the WAXS were related to the presence of crystalline unidentified structures, probably Cho or unknown mixtures containing Cho. Table III shows the summary of the structural parameters. Reconstituted erythrocyte membranes from both groups had a $\mathrm{L}_{\alpha}$ phase with a d-value in the range 6-7 $\mathrm{nm}$. In spite of small differences between the two groups, membranes from obese had a d-value $\sim 0.5 \mathrm{~nm}$ significantly higher $(\mathrm{P}<0.001)$, than the normal-weight subjects, indicating a higher overall membrane width in the obese adolescent subjects. The increase in the repeat spacing value observed must be associated to the sum of the thickness of the lipid bilayer plus the thickness of the water layer between bilayers. 


\section{Discussion}

Despite the amount of data existing on obesity, relatively little information is available concerning adolescent population. Food habits and life style seem to be important factors in the development of obesity in the adolescence. If the diet can induce changes in the lipid profile of cell membrane (Barceló et al. 2009), dietary habits in obese adolescents can affect structural membrane properties and related functions. In this sense, we set out a study to compare the structural properties of the erythrocyte membrane from obese and normal-weight adolescent subjects, assuming that the erythrocyte membrane lipid profile could be a dietary biomarker (Poppitt et al. 2005).

Our data show that the membrane lipid profile in obese adolescents is characterized by an increase in SFA and a decrease in MUFA and n-6 PUFA fatty acids, leading to a significant reduction in the UI that most likely could be a consequence of a diet enriched in saturated fats (Poppitt et al. 2005). Indeed, the phospholipid fatty acid profile was associated with a lower membrane susceptibility to lipid peroxidation compared to normalweight subjects. Among UFA, significant lower linoleic acid content was observed in the membrane of obese subjects that correlated with the level of lipid peroxidation determined by the conjugated dienes and the MDA methods. The level of membrane peroxidation might not be solely attributed to the linoleic acid, also to the presence of conjugated linoleic acid (CLA). However, since the average dietary intake of CLA from food is low (estimated in $0.3 \mathrm{~g} /$ day in Europe according to the EFSA Panel on Dietetic Products, Nutrition and Allergies (NDA), 2010) and dietary CLA intake is poorly incorporated into the membrane phospholipids (Martins et al. 2011), we consider very unlikely that the content of CLA in erythrocyte membrane could significantly alter the levels of conjugated dienes measured, changing the positive correlation observed between linoleic acid and membrane propensity to oxidation. The relevance of linoleic acid in erythrocyte membrane of adolescent subjects is in contrast with a previous study that showed lower n-3 PUFA content and higher oxidative damage in erythrocyte membrane of overweight and obese women (Cazzola et al. 2004). There are scarce data regarding the lipid profile of obese children and adolescents (Warensjo et al. 2006), and mostly correspond to the plasma lipid fraction (Agostoni et al. 1994; Gil-Campos et al. 2008). Some common features reported are an alteration in the linoleic acid metabolism (Reynoso et al. 2003) and a high contribution of n-6 PUFA. Such changes in the lipid profile appear to be an early marker of the metabolic syndrome in children at pre-pubertal age (Gil-Campos et al. 2008). On the other hand, the high levels of palmitic acid found in membrane phospholipids of obese 12 
adolescents might be related to the progression of insulin resistance in these individuals (Reynoso et al. 2003; Haag et al. 2005). Palmitic acid is a substrate for the synthesis of ceramides, which seems to play a role as mediator in lipid-induced insulin resistance (Corcoran et al. 2007) and in the metabolic disease of obesity (Summers et al. 2006). Finally, another significant result was an increase in the Cho content and the Cho/PL ratio in obese adolescents. Cho affects the overall membrane structure and the associated features of fluidity and lipid peroxidation (Cazzola et al. 2004; Ohvo-Rekilä et al. 2002). Structural properties of reconstituted model membranes may be considered as a fingerprint of individual erythrocyte membrane. The obese adolescent group had a significant augment in the membrane d-space value that must be related to changes in the membrane lipid composition in addition to an increase in the water layer between bilayers. Since Cho modulates the membrane lipid order and the lateral packing of PL acyl chains (Ohvo-Rekilä et al. 2002), the raise in the Cho/PL ratio of obese adolescents is expected to reduce the motional order of the phospholipid acyl chains and to increase the membrane-width, as it is shown in this study. Indeed, the increase in the packing density of the acyl chains can promote lipid peroxidation in less fluid environments. In turn, lipid peroxidation can affect the membrane structure by inducing the formation of discrete membrane-restricted Cho domains (Jacob et al. 2005), as we have also observed.

Structural changes in the erythrocyte membrane of obese adolescents can affect membrane functions and have physiological implications. For example, an increase in the Cho/PL ratio may result in morphological adjustments. Changes in the lipid profile affecting the bulk lipid fluidity and membrane structural properties, may have an effect on the organization of specialized membrane lipid domains (Kamata et al. 2008), lipidprotein interactions and the localization and/or activity of membrane-associated proteins (Villar et al. 1996; Mohandas et al. 2008). Several functions related to the AChE activity in the erythrocyte membrane have been described (Lopes de Almeida et al. 2010; Prall et al. 1998; Carvalho et al. 2009). In the context of our work, we can speculate that the lower $\mathrm{AChE}$ activity observed in obese adolescents could be related to a decrease in the enzyme content and/or activity that may also be influenced by protein-interactions in which the membrane structure participates. This part of the work deserves studying other membrane proteins to relate changes in membrane protein function and obesity.

Our present data constitute a first study focused on obese adolescent subjects. The limited number of individuals does not allow us to make generalizations to the whole population of adolescents. But, the study clearly 13 
shows that the consequences of unhealthy dietary habits, such as a high intake of fat and SFA, are reflected in the erythrocyte membrane lipid composition and the structural properties of the reconstituted model membranes. The demonstration that dietary habits have an effect on the erythrocyte membrane properties and may influence the membrane protein activities suggests that such interrelations are of physiological significance and may have implications in the development of diseases associated to the obesity in adolescents.

\section{Acknowledgements}

The authors thank to Dr. Francisco Rivas-Garcia (Ayuntamiento de Guadix) for his assistance in recruiting the participants and providing the infrastructure to conduct the study. We are grateful to Dr. Jesús Prades for technical assistance, Dr. S.S. Funari for helpful discussions regarding the manuscript, Dr. A. Rothkirch for writing A2tool program and making it publicly available and Dra. I. Moreno for the language revision. This work was supported by the "Ayuntamiento de Guadix" (Spain) and funded by Deutsches Elektronen-Synchrotron DESY, HASYLAB (Hamburg, Germany) [proposal II-20090005 EC] and the Spanish Ministry of Economy and Competitiveness [grant AGL2011-23810].

\section{Conflict of interest statement}

The authors declare that there are no conflicts of interest. The authors alone are responsible for the content and writing of the manuscript. 


\section{References}

Agostoni C, Riva E, Bellù R, Vincenzo SS, Grazia BM, Giovannin M (1994) Relationships between the fatty acid status and insulinemic indexes in obese children. Prostaglandins Leukot. Essent. Fatty Acids 51:317-321

Barceló F, Perona JS, Prades J, Funari SS, Gomez-Gracia E, Conde M, Estruch R, Ruiz-Gutiérrez V (2009). Mediterranean-style diet effect on the structural properties of the erythrocyte cell membrane of hypertensive patients: the Prevencion con Dieta Mediterranea Study. Hypertension 54:1143-1150

Carvalho FA, Lopes de Almeida JP, Freitas-Santos T, Saldanha C (2009) Modulation of erythrocyte acetylcholinesterase activity and its association with g protein-band 3 interactions. J. Membr. Biol. 228:89-97

Cazzola R, Rondanelli M, Russo-Volpe S, Ferrari E, and Cestaro B (2004) Decreased membrane fluidity and altered susceptibility to peroxidation and lipid composition in overweight and obese female erythrocytes. J. Lipid Res. 45:1846-1851

Cole TJ, Bellizzi MC, Flegal KM, and Dietz WH (2000) Establishing a standard definition for child overweight and obesity worldwide: international survey. Brit. Med. J. 320: 1240-1243.

Collins CE, Watson J, and Burrows T (2010) Measuring dietary intake in children and adolescents in the context of overweight and obesity. Int. J. Obes. 34: 1103-1115.

Corcoran MP, Lamon-Fava S, and Fielding RA (2007) Skeletal muscle lipid deposition and insulin resistance: effect of dietary fatty acids and exercise. Am. J. Clin. Nutr. 85: 662-677.

Dietz WH (1998) Health consequences of obesity in youth: childhood predictors of adult disease. Pediatrics 101: 518-525.

Ellman GL, Courtney KD, Andres JrV, and Featherstone RM (1961) A new and rapid colorimeteric determination of acetylcholinesterase activity. Biochem. Pharmacol. 7: 88-95.

Escriba PV, Sanchez-Dominguez JM, Alemany R, Perona JS, and Ruiz-Gutiérrez V (2003) Alteration of lipids, G- proteins, and PKC in cell membranes of elderly hypertensives. Hypertension 41: 176-182. 
EFSA Panel on Dietetic Products, Nutrition and Allergies (NDA) (2010) Scientific Opinion on the safety of “conjugated linoleic acid (CLA)-rich oil” (Tonalin TG 80) as a Novel Food ingredient. EFSA J. 8: 1600-1643.

Folch J, Lees M, and Sloane Stanley GH (1957) A simple method for the isolation and purification of total lipides from animal tissues. J. Biol. Chem. 226: 497-509.

Funari SS, Barceló F, Escribá PV (2003) Effects of oleic acid and its congeners, elaidic and stearic acids, on the structural properties of phosphatidylethanolamine membranes. J. Lipid Res. 44: 567-575.

Gil-Campos M, Ramírez-Tortosa MC, Larqué E, Linde J, Aguilera CM, Cañete R, and Gil A (2008) Metabolic syndrome affects fatty acid composition of plasma lipids in obese prepubertal children. Lipids 43: 723-732.

González-Jiménez E, Aguilar-Cordero MJ, García-García CJ, García-López P, Álvarez-Ferre J, Padilla-López CA, and Ocete-Hita E (2012) Influence of family environment of the development of obesity and overweight in a population of school children in Granada (Spain). Nutr Hosp 27: 177-184.

Haag M, and Dippenaar NG (2005) Dietary fats, fatty acids and insulin resistance: short review of a multifaceted connection. Med. Sci. Monit. 11: RA359-RA367.

Jacobsson JA, Rask-Andersen M, Risérus U, Moschonis G, Koumpitski A, Chrousos GP, Lannfelt L, Marcus C, Gyllensten U, Schiöth HB, and Fredriksson R (2012) Genetic variants near the MGAT1 gene are associated with body weight, BMI and fatty acid metabolism among adults and children. Int. J. Obes. 36: 119-129.

Jacob RF, and Mason RP (2005) Lipid peroxidation induces cholesterol domain formation in model membranes. J Biol Chem 280: 39380-39387.

Kamata K, Manno S, Ozaki M, and Takakuwa Y (2008) Functional evidence for presence of lipid rafts in erythrocyte membranes: Gsalpha in rafts is essential for signal transduction. Am. J. Hematol. 83: 371-375.

Lopes de Almeida JP, and Saldanha C (2010) Nonneuronal Cholinergic System in Human Erythrocytes: Biological Role and Clinical Relevance. J. Membr. Biol. 234: 227-234. 
Martins SV, Lopes PA, Alves SP, Alfaia CM, Nascimento M, Castro MF, Bessa RJ, Prates JA (2011) Dietary conjugated linoleic acid isomers change the unsaturation degree of hepatic fatty acids in neutral lipids but not in polar lipids. Nutr. Res. 31:246-254.

Mohandas N, and Gallagher PG (2008) Red cell membrane: past, present, and future. Blood 112: 3939-3948.

Ohvo-Rekilä H, Ramstedt B, Leppimäki P, and Slotte JP (2002) Cholesterol interactions with phospholipids in membranes. Prog. Lipid Res. 41: 66-97.

Pan DA, Hulbert AJ, Storlien LH (1994) Dietary fats, membrane phospholipids and obesity. J. Nutr. 124: 15551565.

Perona JS, and Ruiz-Gutierrez V (2004) Quantification of major lipid classes in human triacylglycerol-rich lipoproteins by high-performance liquid chromatography with evaporative light-scattering detection. J. Sep. Sci. 27: 653-659.

Poppitt SD, Kilmartinv P, Butler P, and Keogh GF (2005) Assessment of erythrocyte phospholipid fatty acid composition as a biomarker for dietary MUFA, PUFA or saturated fatty acid intake in a controlled cross-over intervention trial. Lipids Health Dis 4: 30.

Prall YG, Gambhir KK, and Ampy FR (1998) Acetylcholinesterase: an enzymatic marker of human red blood cell aging. Life Sci. 63: 177-184.

Reilly CA, and Aust SD (1999) Measurement of Lipid Peroxidation in Current Protocols in Toxicology (Maines MD, Costa LG, Reed DJ, Sassa S, and Sipes IG, eds.) pp. 1-13, John Wiley \& Sons Ltd, Oxford.

Reynoso R, Salgado LM, and Calderón V (2003) High levels of palmitic acid lead to insulin resistance due to changes in the level of phosphorylation of the insulin receptor and insulin receptor substrate-1. Mol.Cell Biochem. 246: 155-162.

Ruíz-Gutiérrez V, Muriana FJ, Guerrero A, Cert AM, and Villar J (1996) Plasma lipids, erythrocyte membrane lipids and blood pressure of hypertensive women after ingestion of dietary oleic acid from two different sources. J. Hypertens. 14: 1483-1490. 
Ruiz-Gutierrez V, Montero E, Villar J (1992) Determination of fatty acid and triacylglycerol composition of human adipose tissue. J Chromatogr. 81: 171-178.

Sinha A, and Kling S (2009) A review of adolescent obesity: prevalence, etiology, and treatment. Obes. Surg. 19: $113-120$.

Summers SA (2006) Ceramides in insulin resistance and lipotoxicity. Prog. Lipid Res. 45: 42-72.

Symonds ME, Sebert SP, and Budge, H (2009) The impact of diet during early life and its contribution to later disease: critical checkpoints in development and their long-term consequences for metabolic health. Proc. Nutr. Soc. 68: 416-421.

Villar J, Montilla C, Muñiz-Grijalvo O, Muriana FG, Stiefel P, Ruiz-Gutiérrez V, and Carneado J (1996) Erythrocyte $\mathrm{Na}(+)-\mathrm{Li}+$ countertransport in essential hypertension: correlation with membrane lipids levels. J. Hypertens. 14: 969-973.

Warensjo E, Ohrvall M, and Vessby B (2006) Fatty acid composition and estimated desaturase activities are associated with obesity and lifestyle variables in men and women. Nutr. Metab. Cardiovasc. Dis. 16: 128-136. 


\section{Figure legends}

Fig. 1. Erythrocyte membrane lipid oxidation products. (A) conjugated dienes, (B) malondialdehyde (MDA) levels and (C) correlation between erythrocyte-conjugated dienes and MDA values. Samples: obese $(n=11)$ and normal-weight $(\mathrm{n}=11)$ adolescent subjects. Correlation parameters and significance were calculated with the Pearson correlation method. Data are expressed as mean \pm standard deviation. $* \mathrm{P}<0.05$ obese vs normalweight (unpaired Student's $t$-test).

Fig. 2. Erythrocyte membrane acetylcholinesterase (AChE) activity. Enzyme activity was measured at $37^{\circ} \mathrm{C}$ and is expressed as $\mu \mathrm{mol}$ acetylcholine iodide hydrolysed/min per mg protein. Data are expressed as mean \pm standard deviation. $* \mathrm{P}<0.05$ obese vs normal-weight (unpaired Student's $t$-test).

Fig. 3. (A) A representative experimental scattering pattern of reconstituted erythrocyte membranes at $30{ }^{\circ} \mathrm{C}$. Lines represent ordered scattering from the lamellar liquid crystalline phase $\left(\mathrm{L}_{\alpha}\right)$. (B) Linear plots of the $\mathrm{X}$-ray diffraction patterns of reconstituted membranes: normal-weight (upper panels) and obese (down panels) adolescent subjects. The pattern sequences were acquired after $10 \mathrm{~min}$ sample equilibration at each temperature. Successive diffraction patterns were collected for $15 \mathrm{~s}$. The $\mathrm{L}_{\alpha}$ phase was identified by two diffraction peaks in the SAXS. The peak at $\mathrm{s}=0.289 \mathrm{~nm}^{-1}$ was assigned to a crystalline Cho structure. Diffraction peaks in the WAXS patterns indicate the presence of crystalline structures not identified. 